\title{
Fast Search Techniques for obtaining Space-Time Trellis Codes for Rayleigh Fading Channels and Its Performance in CDMA Systems
}

\author{
Bilal A. Rassool, F. Heliot, L. Revelly, M. Dohler, R. Nakhai, H. Aghvami \\ Centre for Telecommunications Research, King's College London, UK \\ \{bilalrassool, mischa\}@iece.org, \{reza.nakhai, hamid.aghvami\}@kcl.ac.uk
}

\begin{abstract}
In this paper, we aim to obtain optimal spacetime trellis codes and propose novel methods for reducing the highly growing full code search. We show that by exploiting the symmetry in the QAM and PSK constellations, the number of combinations in the generator matrix of the encoder can be halved. We also show that for the same set of columns of the generator matrix, interchanging the columns give identical results hence reducing the full code search by the factorial of the number of transmit antennas. Using the suggested methods, we obtain novel space-time codes for slow Rayleigh fading environments and evaluate their performance by simulation, described by frame error probabilities.

Furthermore, the performance of the obtained novel codes is evaluated in a Multi-User Code Division Multiple Access (CDMA) system.
\end{abstract}

Keywords - Space-time trellis coding, code search, code design criteria, CDMA.

\section{INTRODUCTION}

Multiple Input Multiple Output (MIMO) systems are known to perform better than single link wireless communication systems, by employing multiple transmit, and optionally, multiple receive antennas [1]. Several architectures have been proposed to exploit the potential of MIMO systems, amongst which are space-time trellis coded modulation, space-time block codes and layered space-time architectures. All of these provide high data rates with a given transceiver complexity. With $3 \mathrm{G}$ systems and beyond requiring high data rates for applications such as multimedia, this particular area has gained a lot of research interest.

Barring complexity issues, space-time trellis codes (STTC) have shown to outperform other space-time coded systems. Tarokh introduced the concept of STTC as an extension to the conventional time-convolutional coding, and derived criteria to obtain optimal codes for slow Rayleigh fading channels [2]. The codes are hand derived and not optimal: Optimal codes can only be obtained by considering the code design criteria against all possible combinations of the coefficients in the generator (G-) matrix. However, when constructing codes for high number of transmit antennas with high modulation indices, the full search increases drastically and therefore also increasing the code search time. This may result in a few billion combinations of the generator matrix. For instance, a 4-PSK code with 2 transmit antennas and 16 states has 12 different coefficients in the generator matrix. This implies having $4^{12}$, i.e. $16,777,216$ combinations. The equivalent scenario for a 5-antenna 4-PSK code results in $4^{30}$ combinations in the G-matrix, i.e. $1,152,921,504,606,846,976$ combinations!!

In the following, we propose two novel ways aimed at significantly reducing the code search, but yet obtaining optimal codes. Evaluating the difference matrix [2] or the conjugate of the difference matrix, results in the same parameters of interest in the distance matrix, when satisfying the code design criteria. Hence, in the first method, we scrutinize the symmetry in the constellations and succeed to half the number of combinations when performing a full code search. The second method is based on the mathematical proof that for a particular set of coefficients systematically aligned in the G-matrix, where each column in the G-matrix represents coefficients for a certain transmit antenna, interchanging the columns and performing the encoding operation yields the same results. Hence reducing the code search, by the factorial of the number of transmit antennas under consideration. A significant reduction in the code search can be achieved by combining the above two methods.

The rest of the paper is organised as follows. The system model employed for performing a full code search is presented in Section II. In Section III we introduce the code design criteria for various diversity orders on quasistatic Rayleigh fading channels. Novel ways of reducing the full code search are discussed in-depth in Section IV. In Section V, an example on the effectiveness of the code reduction is presented. In Section VI we present novel code coefficients, obtained using our proposed method for slow Rayleigh fading channels for five and six transmit antennas with 16 and 32 transitional states. The performance of these novel codes are examined by simulation in Section VII, as well as in CDMA systems. Conclusions are given in Section VIII.

\section{System Model for Space-Time Code Search}

We consider a mobile communications system where the transmitter is equipped with $n_{T}$ antennas and a receiver with $n_{R}$ antennas. The signals are assumed to undergo independent flat Rayleigh fading between the transmit and the receive antennas. It is assumed that the path gains are constant during one frame and change from one frame to the other (quasi-static fading).

A two-branch feed-forward shift register with memory order $\nu$ is used to model the 4-PSK STTC encoder with $n_{T}$ transmit antennas [3]. The input to the STTC encoder is a sequence of bits that is divided into two streams at any time $t$, creating $I_{t}^{1}$ and $I_{t}^{2} ; I_{t}^{1}$ being the most significant bit, and $I_{t}^{2}$ the least significant. The two streams of input bits are then simultaneously multiplied by coefficients $\left(a_{p}^{1}, a_{p}^{2}, \ldots, a_{p}^{n_{T}}\right),\left(b_{p}^{1}, b_{p}^{2}, \ldots, b_{p}^{n_{T}}\right)$ respectively, where 
the superscript of the coefficients represents the antenna number and the subscript represents the delay in the encoder, $p=0,1, \ldots \nu_{1}, q=0,1, \ldots \nu_{2}$, with $\nu_{i}=\left\lfloor\frac{\nu+i-1}{2}\right\rfloor$, $i=1,2$, and $a_{p}^{i}, b_{q}^{i} \epsilon\{0,1,2,3\}$ for 4-PSK modulation. The resulting data is added in modulo 4 .

This encoding operation can be expressed in matrixmultiplication form $\mathbf{x}_{t}=\mathbf{I} \cdot \mathbf{G}$ where $\mathbf{x}_{t}=\left(x_{t}^{1}, x_{t}^{2}, \ldots, x_{t}^{n_{T}}\right)$ is the output from each antenna $x^{i}, i=1,2, \ldots n_{T}$, at time $t, \mathbf{I}$ is the input bit-vector and $\mathbf{G}$ is defined as the generator matrix, where each of its columns represents the coefficients for -each transmit antenna. Here, the coeffcients in the G-matrix are arranged in an alternative form, such that for a 4 -state code with 5 antennas it would be represented as:

$$
\mathbf{G}=\left(\begin{array}{cccc}
a_{0}^{1} & b_{0}^{1} & a_{1}^{1} & b_{l}^{1} \\
a_{0}^{2} & b_{0}^{2} & a_{1}^{2} & b_{l}^{2} \\
a_{0}^{3} & b_{0}^{3} & a_{1}^{3} & b_{l}^{3} \\
a_{0}^{4} & b_{0}^{4} & a_{1}^{4} & b_{l}^{4} \\
a_{0}^{5} & b_{0}^{5} & a_{1}^{5} & b_{l}^{5}
\end{array}\right)
$$

\section{Code Design Criteria for Quasi-Static Rayleigh Fading Channels}

Having transmitted codeword $\mathrm{c}_{t}=\left(c_{t}^{1}, c_{t}^{2}, \ldots, c_{t}^{n T}\right)$, an error occurs when the decoder makes an erroneous decision and chooses $\mathbf{e}_{t}=\left(e_{t}^{1}, e_{t}^{2}, \ldots, e_{t}^{n_{T}}\right)$ as the most likely codeword. The difference matrix $B$ is then represented as the corresponding codewords $\mathbf{c}$ and $\mathrm{e}$ :

$$
\mathbf{B}(\mathbf{c}, \mathbf{e})=\left(\begin{array}{cccc}
e_{0}^{1}-c_{0}^{1} & e_{1}^{1}-c_{1}^{1} & \ldots & e_{L}^{1}-c_{L}^{1} \\
e_{0}^{2}-c_{0}^{2} & e_{1}^{2}-c_{1}^{2} & \ldots & e_{L}^{2}-c_{L}^{2} \\
\vdots & \vdots & \ddots & \vdots \\
e_{0}^{n_{T}}-c_{0}^{n_{T} T} & e_{1}^{n_{T}}-c_{1}^{n_{T}} & \ldots & e_{L}^{n_{T}}-c_{L}^{n_{T}}
\end{array}\right)
$$

The distance matrix $\boldsymbol{A}$, is the Euclidean distance be tween the corresponding codewords transmitted at each time from each transmit antenna $n_{T}$, and the erroneous codeword. It is obtained by performing the following:

$$
\mathbf{A}=\mathbf{B} \cdot \mathbf{B}^{H}
$$

where $H$ denotes the Hermitian (transpose conjugate).

For slow Rayleigh fading channels, when the diversity order of the system is three or less, where the diversity order is defined as the product of the number of transmit and receive antennas, an upper bound on the pairwise error probability is given by [2], [3]:

$$
P(\mathbf{c} \rightarrow \mathbf{e}) \leq\left(\prod_{i=1}^{r} \lambda_{i}\right)^{-n_{R}}\left(\frac{E_{s}}{4 N_{o}}\right)^{-r n_{R}}
$$

where $\lambda_{i}$ are the eigenvalues and $r$ the rank of the distance matrix $\boldsymbol{A}$ (explained in IV), $n_{R}$ is the number of receive antennas, $E_{s}$ is the energy per symbol at each transmit antenna, and $N_{o}$ is the noise spectral density. Hence, we may optimise the coding gain by maximising the minimum determinant of the distance matrix, obtained over all combinations of distinct codewords. When the diversity order of the system is four or more, the upper bound on the minimum pairwise error probability is given by [5]

$$
P(\mathrm{c} \rightarrow \mathrm{e}) \leq \frac{1}{2} \exp \left(-n_{R} \frac{E_{s}}{4 N_{o}} \sum_{i=1}^{n_{T}} \lambda_{i}\right)
$$

Hence, we may optimise the coding gain by maximising the trace of the distance matrix, $\mathbf{A}$.

\section{Reducing the Code Search}

\section{A. Novel Code Search Criteria: Method 1}

The first method for reducing the code search whilst obtaining optimal codes is based on the fact that when performing a full search with all possible codewords and all possible Error Event Paths (EEPs) for each set of coefficients, we duplicate the search by evaluating the mirror image of the difference matrix, i.e., we make calculations on the complex conjugate of the difference matrix. This is because for the same set of coefficients, we interchange with the codeword being the EEP and vice versa, and effectively do the same calculations on $B$ and $B^{*}$. All possible combinations of the codeword and EEP have to be taken into account due to the geometrical non-uniformity of codes [6]. We now ensure that reducing the code search in such a way does not affect obtaining optimal codes.

Theorem 1: Satisfying the criteria for obtaining optimal STTC from $B$, or the conjugate of $B$, i.e., $B^{*} ;$ results in the same values of interest from the distance matrix $\boldsymbol{A}$.

Proof: Considering the complex conjugate of $\mathrm{B}^{*}$, we obtain the following using (3)

$$
\mathbf{A}^{*}=\left(\mathbf{B} \cdot \mathbf{B}^{H}\right)^{*}
$$

$\boldsymbol{A}$ is Hermitian [2], therefore we obtain the same rank, same eigenvalues and same determinant of $\boldsymbol{A}$ and $\boldsymbol{A}^{*}$. Since the trace is simply the sum of the eigenvalues [7], both design criteria for slow fading channels are unaffected by this code reducing method.

\section{B. Novel Code Search Criteria: Method 2}

When performing a code search on the coefficients in the G-matrix, where each column represents the coefficients for a particular transmit antenna, we effectively repeat the columns of the G-matrix but in a different order. Hence, we can reduce the full code search by the factorial of the number of columns, i.e., number of transmit antennas, as long as it does not affect the parameters of interest when satisfying the criteria for obtaining optimal space-time codes for both slow and fast fading environments. This is proven in the following theorem.

Theorem 2: Interchanging the rows of the difference matrix does not influence the criteria for obtaining optimum STTC, and results in the same values of interest from the $A$ matrix.

Proof : Consider swapping the first and second columns of the G-matrix, this implies interchanging first two rows of the $B$ matrix, which results in, say, $\mathbf{B}^{\prime}$ 


$$
\mathbf{B}^{\prime}(\mathbf{c}, \mathbf{e})=\left(\begin{array}{cccc}
e_{0}^{2}-c_{0}^{2} & e_{1}^{2}-c_{1}^{2} & \ldots & e_{l}^{2}-c_{l}^{2} \\
e_{0}^{1}-c_{0}^{1} & e_{1}^{1}-c_{1}^{1} & \ldots & e_{l}^{1}-c_{l}^{1} \\
\vdots & \vdots & \ddots & \vdots \\
e_{0}^{n_{T}}-c_{0}^{n_{T}} & e_{1}^{n_{T}}-c_{1}^{n_{T}} & \ldots & e_{l}^{n_{T}}-c_{l}^{n_{T}}
\end{array}\right)
$$

where $B^{\prime}$ is a permutation of $B$ along the rows. The same principle holds for $\left(\boldsymbol{B}^{H}\right)^{\prime}$ and $\boldsymbol{B}^{H}$ but along the columns, since Hermitian includes a transpose. When calculating the distance matrix $\mathbf{A}^{\prime}$, the multiplication is commutative and we obtain the following

$$
\mathbf{A}^{\prime}=\left(\mathbf{B} \cdot \mathbf{B}^{H}\right)^{\prime}
$$

where $\mathbf{A}^{\prime}$ is a permutation of $A$ along the rows and the columns.

We now look at how these changes in the distance matrix $\mathbf{A}^{\prime}$ affect the parameters of interest when constructing codes. Firstly, we evaluate the determinant of $\mathbf{A}^{\prime}$. Swapping, once only, any two rows or columns of matrix results in the same determinant multiplied by -1 [7], hence

$$
\operatorname{det} \mathbf{A}=(-1)^{n} \operatorname{det}\left(\mathbf{A}^{\prime}\right)
$$

where $n$ is the number of permutations and $n \in N$.

Since there are $k$ permutations on the columns of $\mathbf{G}$, which result in $k$ permutations on the rows of $B$. When evaluating $\boldsymbol{B}^{H}$, we inevitably make $k$ permutations on its columns. Therefore, $\boldsymbol{A}$ and $\mathbf{A}^{\prime}$ will always differ from each other by $2 k$ permutations. Hence, $n$ is always even and the following always holds:

$$
\operatorname{det} \mathbf{A}=\operatorname{det}\left(\mathbf{A}^{\prime}\right)
$$

'Furthermore, any two matrices differing by two interchanged rows or columns have the same rank.

When the diversity order of the system is 4 or more, we evaluate the trace of the distance matrix $\boldsymbol{A}$. The trace may be expressed as follows

$$
\operatorname{tr}(\mathbf{A}(\mathbf{c}, \mathbf{e}))=\sum_{i=1}^{n_{T}} \lambda_{i}=\sum_{i=1}^{n_{T}} A_{i i}=\sum_{i=1}^{n_{\gamma}} \sum_{j=1}^{l}\left|c_{j}^{i}-e_{j}^{i}\right|^{2}
$$

Interchanging any two rows or columns of a matrix simply alters the order of the summation required to evaluate the trace [7]. Hence, evaluating the trace of $\boldsymbol{A}$ or $\mathbf{A}^{\prime}$ give the same result.

\section{Comparing the Methods for Reducing Full Code Search}

In the following we consider an example to compare the code-search reduction methods. To obtain codes for a 3 antenna, 4-state, 4-PSK code via a full search, there are $4^{12}=16,777,216$ combinations in the generator matrix. Using the known sub-optimal method [4], we first do a full search on the 2-antenna, 4-PSK, 4-state case, which has $4^{8}=65,536$ combinations; and then a full search on the added 4 coefficients that has $4^{4}=256$ combinations. Hence the total number of combinations are $4^{8}+4^{4}=$ 65,792 combinations.

Using the first method proposed here, which halves the code search, we have $\frac{4^{12}}{2}=8,388,608$ combinations. With the second proposed method, the code search is reduced by the factorial of the number of antennas, and we carry out the search on $\frac{4^{12}}{3 !}=2,796,203$ combinations of $\mathbf{G}$. Combining the two novel methods results in $1,398,101$ combinations. This number is significantly lower than the number of full code search combinations but higher than the suboptimal method; however it guarantees obtaining optimum space-time trellis codes.

\section{Novel Space-time Codes Obtained Using the Reduced Search Criteria}

Using a combination of our proposed methods, new 4PSK codes are obtained. In Table I below, STTCs codes for 16 and 32 transitional states for slow fading channels, and varying number of transmit antennas are reported. The coefficients in the tables represent the G-matrix and are read as in (1).

\begin{tabular}{|c|c|c|c|}
\hline $5 T x 16 S$ & $6 T x 16 S$ & $5 T \times 32 S$ & $6 T \times 32 S$ \\
\hline 21321 & 132122 & 22220 & 120222 \\
02212 & 220213 & 13202 & 222031 \\
13202 & 231200 & 22112 & 332322 \\
22020 & 022022 & 30223 & 121210 \\
21123 & 312122 & 01223 & 221203 \\
02232 & 220231 & 30132 & 032110 \\
& & 12002 & 302032 \\
\hline Rank 2 & 2 & 3 & 3 \\
\hline Trace 40 & 48 & 44 & 52 \\
\hline
\end{tabular}

TABLE I

COEFFICIENTS FOR 4-PSK, 16- AND 32-STATES CODES FOR SLOW FADING CHANNELS, WITH FIVE AND SIX TRANSMIT ANTENNAS.

\section{ViI. Code Performance}

The code performance is evaluated by simulation and described by frame error rate (FER). Each frame consists of 130 symbols transmitted from each transmit antenna, and ideal channel state information is assumed at the receiver. The performance curves are plotted against the signal-tonoise ratio (SNR) per receive antenna.

The simulation results in Fig. 1 show that the codes for 16-states with $n_{T}=6$ are superior to the ones of $n_{T}=5$, and the codes with $n_{T}=5$ have better performance compared to the corresponding ones in [4]. An improvement of around 0.9 arid $0.6 \mathrm{~dB}$ is observed at the FER of $10^{-2}$ when $n_{T}$ is increased from four to five, and from five to six respectively. Correspondingly, less gains in performance are observed when increasing the number of receive anten-. nas. The 4-antenna code is from [4] and is included for reference only. Similar observations are observed for the 32 -state codes in 2 


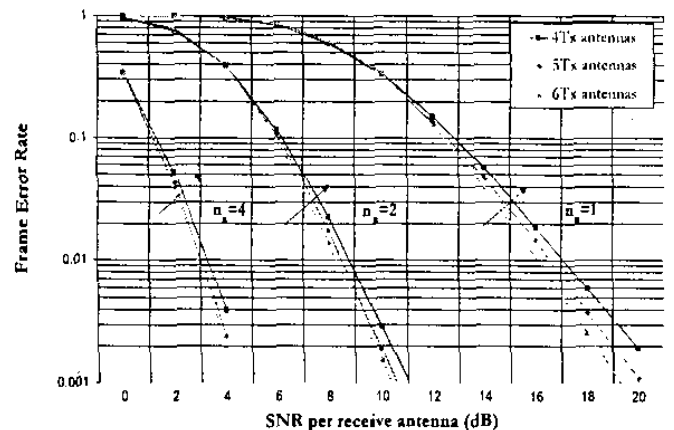

Fig. 1. Performance comparison of the 16-state STTC with 4, 5 and 6 transmit antennas on slow fading channels.

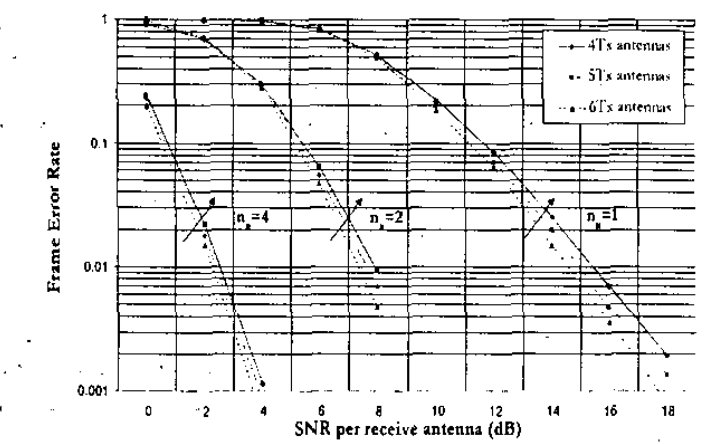

Fig. 2. Performance comparison of the 32 -state STTC with 4,5 and 6 transmit antennas on slow fading channels.

In Fig. 3, the FER performance in a synchronous CDMA - system is evaluated by simulations. In accordance with $[8]$, the frame length is 150 symbols from each transmit antenna with a spreading factor of 15 . Perfect channel state information is assumed at the receiver. Note that the results from [8] is for 2 transmit antennas with 4 -states whereas these results are for 16 states, hence making the comparison not entirely fair, but still informative. Results show that the FER performance is best with 5 transmit antennas.

Fig. 3 also depicts the performance of 16-state QPSK codes with 4 transmit antennas and 1 receive antenna and various co-channel user in CDMA systems. Results show worsening behaviour with increasing number of users, as expected.

\section{CONClusions}

'Different ways of reducing the space-time trellis code search has been investigated in this paper. We propose two novel methods for obtaining codes that best satisfy the spacetime code design criteria. The novel methods are verified mathematically, and when used together can reduce the code search by twice the factorial of the number of transmit antennas under consideration.

In the process, novel codes are obtained for varying num-

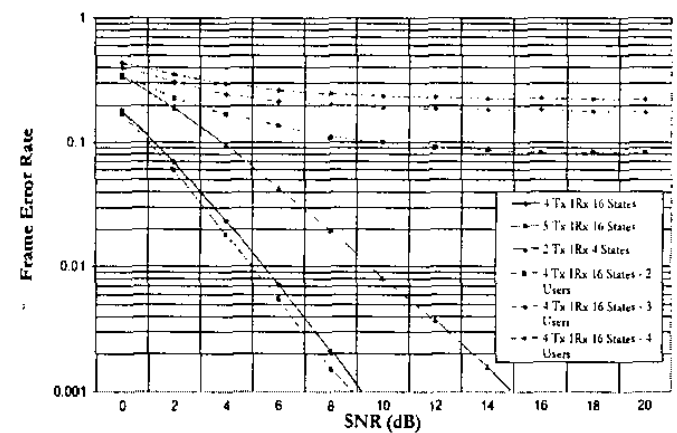

Fig. 3. Performance comparison a multiuser CDMA system with 16state STTC with 2,3 and 4 users, 4 transmit and 1 receive antenna. For 1 user, performance comparison is drawn for 2,4 and 5 transmit antennas with 1 receive.

ber of transmit antennas and number of states, and their performance is evaluated by considering frame error probabilities. Also the performance of the obtained codes is observed in a multi-user CDMA system.

\section{ACKNOWLEDGEMENTS}

The work reported in this paper has formed part of the Wireless Access area from the Core 2 Research Programme of the Virtual Centre of Excellence in Mobile \& Personal Communications, Mobile VCE, www.mobilevce.co.uk. Their funding is gratefully acknowl edged. More detailed information on this research is available to Industrial Members of Mobile VCE.

\section{REFERENCES}

[1] E. Telatar, Capacity of Multi-antenna Gaussian Channels, European Transactions on Telecommunications, Vol. 10, No. 6 Nov. 1999.pp 585-595.

[2] V. Tarokh, N. Seshadri, A. Calderbank, Space-Time Codes for High Data Rate Wireless Communication: Performance Criterion and Code Construction, IEEE Transactions on Info. Theory, Vol.44, pp. 1744-1765, March 1998.

[3] Z. Chen, J. Yuan, B. S. Vucetic, Improved space-time trellis coded modulation on slow Rayleigh fading channels, Electronics Letters, Vol.37, No.7, pp 440-441 March 2001.

[4] Z. Chen, B. S. Vucetic, J. Yuan, K. L. Lo, Space-time trellis codes for 4-PSK with three and four transmit antennas in quasistatic flat fading channels, IEEE Comm Letters, Vol.6, No.2, pp. 67.69, February 2001.

[5] J. Yuan, Z. Chen, B. Vucetic and W. Firmanto, Performance analysis and design of space-time codes on fading channels, accepted for publication in IEEE Transactions on Communications.

[6] J. D. Forney Jr., Geometrically Uniform Codes, IEEE Trans. on Information Theory, Vol.37, No.5 September 1991, pp.1241 1260 .

[7] E. Kreyszig, Advanced Engineering Mathematics, John Wiley Sons, 1993

[8] J. Yuan, B. Vucetic, B. Xu, Z. Chen, Design of Space-time Codes and Its Performance in CDMA Systems, Proc, of IEEE VTC Spring 2001. 